Revista peruana de biología 26(1): 095 - 100 (2019) doi: http://dx.doi.org/10.15381/rpb.v26i1.15912 ISSN-L 1561-0837; elSSN: 1727-9933 Universidad Nacional Mayor de San Marcos

\section{Caracterización química y actividad antimicrobiana del aceite esencial de las hojas de Libanothamnus neriifolius (Asteraceae)}

Chemical characterization and antimicrobial activity of the essential oil of the leaves of Libanothamnus neriifolius (Asteraceae)

\section{Rosa Aparicio-Zambrano ${ }^{1 *}$, Luis Rojas-Fermín ${ }^{1}$, Judith Velasco ${ }^{2}$, Alfredo Usubillaga ${ }^{1}$, Marcos Sosa ${ }^{3}$, Julio Rojas ${ }^{4}$}

Facultad de Farmacia y Bioanálisis, Universidad de Los Andes, Mérida. Venezuela.

$\begin{array}{ll}\text { Presentado: } & 07 / 08 / 2018 \\ \text { Aceptado: } & 26 / 12 / 2018 \\ \text { Publicado online: } & 18 / 03 / 2019\end{array}$

Correspondencia:

1 Instituto de Investigaciones, Dr. Alfredo Nicolas Usubillaga del Hierro", Sección Productos Naturales, Facultad de Farmacia y Bioanálisis, Universidad de Los Andes. Mérida. Venezuela.

2 Laboratorio de Síndromes Gastrointestinales y Urinarios (SGU) "Profa. Luisa Vizcaya", Departamento de Microbiología y Parasitología, Facultad de Farmacia y Bioanálisis, Universidad de Los Andes. Mérida. Venezuela.

3 Escuela de Bioanálisis, Facultad de Farmacia y Bioanálisis, Universidad de Los Andes. Mérida. Venezuela.

4.- Departamento de Toxicología, Facultad de Farmacia y Bioanálisis, Universidad de los Andes. Mérida. Venezuela.

*Autor de correspondencia, dirección postal: 5101

Email RLAZ: rosaaparicio@ula.ve

Email LR-F: rojasfermin33@gmail.com

Email JV: judivel@ula.ve

Email AU: usubi80@gmail.com

Email MS: marquitoss5@gmail.com

Email JR: juliorojas@ula.ve

Citación:

Aparicio-Zambrano R., L. Rojas-Fermín, J. Velasco, A. Usubillaga, M. Sosa, J. Rojas. 2019. Caracterización química y actividad antimicrobiana del aceite esencial de las hojas de Libanothamnus neriifolius (Asteraceae). Revista peruana de biología 26(1): 095 - 100 (Febrero 2019). doi: http://dx.doi. org/10.15381/rpb.v26i1.15912

Palabras clave: Aceite esencial; Libanothamnus neriifolius; actividad antimicrobiana.

Keywords: Essenthial oils; Libanothamnus neriifolius; antimicrobial activity.

\begin{abstract}
Resumen
El aceite esencial de las hojas frescas de Libanothamnus neriifolius (B. ex H) Ernst., fue obtenido por el método de hidrodestilación utilizando la trampa de Clevenger, obteniendo $1.8 \mathrm{~mL}$ (rendimiento $0.087 \%$ ). El aceite esencial se caracterizó por el método de cromatografía de gases acoplado a espectrometría de masas (CG-EM), identificando como compuestos principales 6 -felandreno (29.04\%), $\alpha$-felandreno (19.86\%), $\alpha$-pineno (13.57\%) y $\alpha$-tujeno (12.35\%). La actividad antimicrobiana se determinó por el método de difusión en agar con discos, frente a bacterias y levaduras de referencia internacional (Staphylococcus aureus ATCC 25923, Enterococcus faecalis ATCC 29212, Escherichia coli ATCC 25922, Klebsiella pneumoniae ATCC 23357, Pseudomonas aureginosa ATCC 27853, Candida albicans CDC-B385, Candida krusei ATCC 6258). El aceite esencial inhibió el desarrollo de S. aureus, C. albicans y C. Krusei, con un valor de Concentración Inhibitoria Mínima (CIM) de $50 \mu \mathrm{L} / \mathrm{mL}$, $700 \mu \mathrm{L} / \mathrm{mL}$ y $500 \mu \mathrm{L} / \mathrm{mL}$, respectivamente. Este es el primer reporte sobre actividad antimicrobiana para L. neriifolius y para el género Libanothamnus.
\end{abstract}

\section{Abstract}

The essential oil of the fresh leaves of Libanothamnus neriifolius (B. ex H) Ernst. was obtained by the hydrodistillation method using the Clevenger trap, obtaining $1.8 \mathrm{~mL}(0.087 \%$ yield). The essential oil was characterized by the method of gas chromatography coupled to mass spectrometry (GC/MS), identifying as main compounds, that 6 -phelandrene $(29.0 \%), \alpha$-phelandrene $(19.9 \%), \alpha$-pinene $(13.6 \%)$, and $\alpha$-tujene (12.4\%) were the most abundant constituents. Antimicrobial activity of the oil was tested Staphylococcus aureus ATCC25923, Enterococcus faecalis ATCC 29212, Escherichia coli ATCC 25922, Klebsiella pneumoniae ATCC 23357, Pseudomonas aureginosa ATCC 27853, Candida albicans CDC-B385, Candida krusei ATCC 6258 by the agar difusion method. It showed activity against $S$. aureus with MIC of $50 \mu \mathrm{L} / \mathrm{mL}$. With respect to antifungic activity it was active against $C$. albicans and C. krusei with MIC of $700 \mu \mathrm{L} / \mathrm{mL}$ and $500 \mu \mathrm{L} / \mathrm{mL}$ respectively. This is the first report on the antimicrobial activity of this L. neriifolius and for the genere Libanothamnus. 


\section{Introducción}

En la familia Asteraceae se reconocen un gran número de especies (23000), con una amplia distribución en todo el planeta, convirtiendo a esta familia en una fuente de recursos alternativos y renovables para la humanidad, siendo muchas de sus especies utilizadas en agricultura, industria, medicina, comercio, entre otras (Izco 2004).

La subtribu Espeletiinae, descrita por Cuatrecasas (1976), es un grupo representativo de la familia Asteraceae, conformado por ocho géneros (Carramboa, Espeletiopsis, Coespeletia, Espeletia, Libanothamnus, Ruilopezia, Tamania y Paramiflos) y 146 especies conocidas como frailejones, inciensos, trementinos o tabaqueros; sus especies se extiende desde la cordillera de Mérida, Táchira y Trujillo en Venezuela, en las 3 cordilleras en Colombia, y la parte media y norte de Ecuador (Cuatrecasas 1976).

Libanothamnus neriifolius (B. ex H) Ernst. es conocida como frailejón o tabacote, distribuida principalmente en los Andes venezolanos, no obstante en Colombia se presentan poblaciones aisladas en el norte de la Sierra de Perijá y en la Sierra Nevada de Santa Marta, entre los 1500 y $1800 \mathrm{~m}$ de altitud (Schnee 1960).

Los aceites esenciales de especies de Espeletiinae son usados en la medicina tradicional en afecciones respiratorias, bronquitis, gripe, tos, asma y problemas digestivos, entre otras (Giraldo et al. 2009, López-Palacios 1984, García-Barriga 1975, Otaiza 1999, Albornoz 1997). Sin embargo, es importante resaltar las escasas investigaciones de estas especies y principalmente de L. neriifolius. En tal sentido, Usubillaga et al. (2001) caracterizaron a partir del aceite esencial los constituyentes volátiles de las hojas de cuatro especies de Libanothamnus procedentes de los Andes venezolanos: de L. neriifolius, del Páramo del Batallón, encontraron los siguientes compuestos mayoritarios: sabineno (25.6\%), limoneno (18.6\%) y $\alpha$-tujeno (18.1\%), con un rendimiento de $0.013 \%$ y un total de $95.9 \%$ de identificación de los componentes del aceite esencial extraído. Así mismo, de L. humbertii, recolectado de la Laguna Negra, se identificaron limoneno (19.40\%), $\alpha$-tujeno (18.10\%), $\alpha$-felandreno (18.50\%). De L. lucidus procedente de la segunda estación del Teleférico, La Aguada, se identificaron $\alpha$-tujeno (30.20\%), $\alpha$-pineno (22.20\%), $\alpha$-felandreno (18.50\%). Por último, de $L$. occultus recolectado en el Páramo del Batallón, los componentes mayoritarios fueron el $\alpha$-pineno (31.30\%) Mirceno (17.10\%), $\beta$-pineno (13.00\%). En las cuatro especies estudiadas, los hidrocarburos monoterpénicos fueron los más abundantes. Por otra parte, en el género Libanothamnus se reporta la presencia de diterpenos tipo kaureno que son interesantes tanto desde el punto de vista químico como farmacológico (Bohlmann et al. 1980, Badillo 2001, Khouri et al. 2000).

Con la finalidad de contribuir con el estudio químico y actividad biológica del género Libanothamnus, en el presente estudio se caracterizó el aceite esencial obtenido de las hojas de la especie L. neriifolius y se evaluó su actividad antimicrobiana por el método de difusión en agar con discos frente a bacterias y hongos de referencia internacional.

\section{Material y métodos}

Recolección del material vegetal.- Las hojas frescas de $L$. neriifolius, fueron recolectadas en la carretera vía San José de Acequias a $10 \mathrm{~km}$ de la población, Municipio Campo Elías del Estado Mérida, a una altitud de $2500 \mathrm{~m}$. El género y especie fue identificado por el profesor Pablo Meléndez y el Ing. Juan Carmona, adscritos al Herbario de la Facultad de Farmacia y Bioanálisis, un voucher № R.A. 01, fue depositado en el herbario MERF Ruíz Terán de la Facultad de Farmacia y Bioanálisis de la Universidad de Los Andes.

Extracción del aceite esencial.- Para la extracción del aceite esencial se utilizaron las hojas frescas (2050 g) de L. neriifolius, las cuales fueros sometidas a un proceso de hidrodestilación utilizando la trampa Clevenger. Al aceite obtenido se le adicionó sulfato de sodio anhídrido para eliminarle la humedad, fue almacenado a $4{ }^{\circ} \mathrm{C}$ resguardado de la luz y el oxígeno.

Cromatografía de Gases Acoplada a Espectrometría de Masas (CG-EM).- Para la separación e identificación de los componentes del aceite esencial de la hojas de L. neriifolius, se empleó un cromatógrafo de gases marca Heweltt Packard modelo 6890 con una columna de fenil-metil-polixilosano (HP-5 MS), además un detector de masa marca Heweltt Packard MSD 5973. Se preparó una solución al 2\% (20 $\mu \mathrm{L}$ del aceite en 1 $\mathrm{mL}$ de éter dietílico) y se inyectó $1 \mu \mathrm{L}$ en el equipo, el tiempo del análisis fue de 50 minutos. Con un programa de temperatura inicial de $60{ }^{\circ} \mathrm{C}$, siguiendo intervalos de aumento de $4{ }^{\circ} \mathrm{C} / \mathrm{min}$ hasta una temperatura final de $260{ }^{\circ} \mathrm{C}$ con un split de 100:1. La caracterización e identificación de los compuestos obtenidos se realizó utilizando la base de datos Wiley MS Data Library 6th edición (Adams 2007).

Cálculo de los Índices de Kováts.- Se determinaron los índices utilizando una columna de metil-fenil-silicona de 30 metros y $0.25 \mathrm{~mm}$ de diámetro utilizando una serie de patrones de $n$-parafinas desde la $\mathrm{C}_{7}$ hasta la $\mathrm{C}_{22}$ (Kováts 1958).

Evaluación de la actividad antimicrobiana del aceite esencial.- La actividad antibacteriana se realizó por el método de difusión en agar con discos descritos por Velasco et al. (2007), y la actividad antifúngica por el mismo método de acuerdo a Contreras et al. 2016. Adicionalmente en los ensayos antimicrobianos se colocó un disco estándar del antibiótico y antimicótico de referencia como control positivo (Tabla 2).

Determinación de la concentración inhibitoria mínima (CIM).- Solo se determinó la CIM contra los microorganismos que mostraron susceptibilidad al aceite esencial. Para la determinación de la CIM se prepararon diluciones del aceite esencial con dimetilsulfóxido (DMSO) en un rango de concentración de 10-900 $\mu \mathrm{L} /$ $\mathrm{mL}$, y se impregnaron discos con $10 \mu \mathrm{L}$ de cada dilución. La CIM fue definida como la concentración más baja capaz de inhibir el crecimiento del microorganismo visible (CLSI 2016). Los ensayos se realizaron por duplicado. 


\section{Resultados y discusión}

Del proceso de hidrodestilación de las hojas frescas de L. neriifolius se obtuvo $1.8 \mathrm{~mL}$ del aceite esencial para un rendimiento de $0.087 \%$, con las siguientes características: aspecto transparente, olor característico y color verde claro.

En la Tabla 1 y Figura 1 (cromatograma general), se muestran los compuestos identificados del aceite esencial con sus índices de Kováts de tabla y los calculados, además del porcentaje de área.

El análisis CG-EM, reveló que el aceite esencial obtenido de las hojas de L. neriifolius, contiene 19 compuestos químicos, entre los cuales se destacan por su abundancia el $\beta$-felandreno con $29.04 \%$, $\alpha$-felandreno con $19.86 \%$, $\alpha$-pineno con $13.57 \%$ y el $\alpha$-tujeno con $12.3 \%$. Los componentes volátiles identificados representan el 90.73\% del total del aceite estudiado, porcentaje que difiere del reportado por Usubillaga et al. (2001) donde lograron la identificación de compuestos en un 95.9\% del total del aceite esencial.

En relación a los componentes no identificados (NI) a través de las bases de datos Wiley y NIST representa un $7.88 \%$, lo que indica la presencia de nuevos componentes en el aceite esencial de las hojas de L. neriifolius, incluso del género, en tal sentido se requieren futuras investigaciones para dilucidar los compuestos mencionados y contribuir de esta manera con el estudio fitoquímico de esta especie en particular.

En relación a la naturaleza de los compuestos del aceite esencial de las hojas de L. neriifolius, se observa un predominio de hidrocarburos monoterpénicos (87.28\%), seguido de monoterpenos oxigenados (1.49\%) y sesquiterpenos (1.69\%), resultados similares a lo reportado para esta especie por Usubillaga et al. (2001). Al comparar los tipos de compuestos mayoritarios de nuestros resultados con los de Usubillaga et al. (2001), se observan semejanzas,

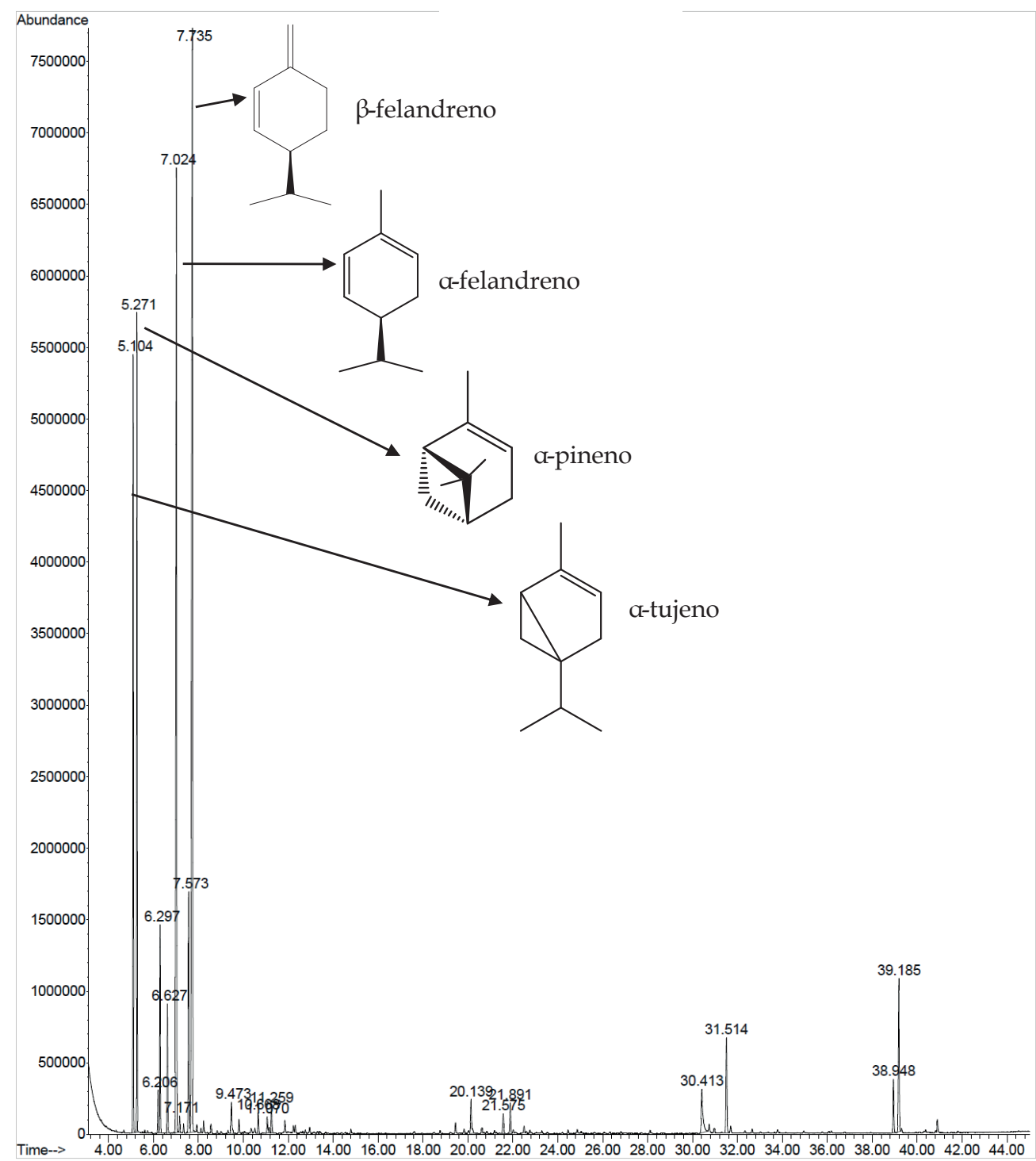

Figura 1. Cromatograma general y compuestos mayoritarios del análisis del aceite esencial de Libanothmnus neriifolius (B. ex H) Ernst. 
Tabla 1. Componentes identificados en el aceite esencial obtenido de las hojas de Libanothamus neriifolius (B. ex H) Ernst.

\begin{tabular}{|c|c|c|c|c|c|}
\hline $\mathbf{N}^{\circ}$ Pico & T.R. & \% Área & NOMBRE DEL COMPUESTO & I.K. Tab. & I.K. Calc. \\
\hline 1 & 5.12 & 12.35 & $\alpha$-tujeno & 930 & 918 \\
\hline 2 & 5.27 & 13.57 & $\alpha$-pineno & 939 & 925 \\
\hline 3 & 6.20 & 0.71 & Sabineno & 975 & 958 \\
\hline 4 & 6.29 & 3.50 & B-pineno & 979 & 961 \\
\hline 5 & 6.62 & 2.17 & Mirceno & 990 & 972 \\
\hline 6 & 7.03 & 19.86 & $\alpha$-felandreno & 1002 & 984 \\
\hline 7 & 7.17 & 0.29 & $\Delta$-3-careno & 1011 & 988 \\
\hline 8 & 7.57 & 5.03 & o-cimeno & 1026 & 1000 \\
\hline 9 & 7.74 & 29.04 & B-felandreno & 1029 & 1007 \\
\hline 10 & 9.47 & 0.76 & $\alpha$-terpinoleno & 1088 & 1080 \\
\hline 11 & 10.67 & 0.46 & $\alpha$-canfolenal & 1126 & 1123 \\
\hline 12 & 11.07 & 0.41 & Trans-pinocarveol & 1139 & 1137 \\
\hline 13 & 11.26 & 0.62 & Trans-verbenol & 1144 & 1143 \\
\hline 14 & 20.14 & 0.95 & B-cariofileno & 1408 & 1423 \\
\hline 15 & 21.89 & 0.74 & $\alpha$-amorfeno & 1484 & 1484 \\
\hline 16 & 30.42 & 1.91 & 139 (100 \%), 109 (49.7 \%), 276 (43.4 \%), 191 (22.2 \%) PM: 276 & $\mathrm{NI}$ & 1783 \\
\hline 17 & 31.51 & 2.57 & 139 (100 \%), 278 (18.8 \%), 153 (12.4 \%), 140 (12.2 \%) PM: 278 & $\mathrm{NI}$ & 1828 \\
\hline 18 & 39.18 & 3.40 & 167 (100 \%), 109 (39.8 \%), 306 (26.8 \%), 191 (23.5 \%) PM: 306 & $\mathrm{NI}$ & 2144 \\
\hline 19 & 40.90 & 0.27 & Kaurenal* & 2244 & 2233 \\
\hline
\end{tabular}

T. R: Tiempo de retención de los componentes. \% Área: área relativa. IK $K_{\text {ref }}$ Índice de Kováts tomado de la bibliografía (Adams. 2007). IK calc Índice de Kováts calculado con la temperatura programada en la columna HP-5 MS. PM: Peso molecular; NI: No identificado (Wiley, NIST).

*Identificado por comparación directa con una muestra autentica.

sin embargo los porcentajes varían, además en nuestra investigación se identificó $\beta$-felandreno, esta diferencia podría atribuirse a cambios en las condiciones físicas (clima, temperatura, depredadores, variaciones fisiológicas, y factores genéticos, entre otros) que contribuyeron con la evolución de esta especie para adaptarse y poder sobrevivir en el medio ambiente que la rodea (Figueiredo et al. 2008). Por otra parte Gobbo-Neto y Lopes (2007) estudiaron en plantas medicinales los siguientes factores que influyen en el contenido de metabolitos secundarios: estado, años, temperatura, agua, radiación UV, nutriente del suelo, altitud, composición atmosférica, entre otros.

En cuanto a la actividad biológica, el aceite esencial de las hojas de L. neriifolius mostró acción antibacteriana solo contra Staphylococcus aureus ATCC 25923, con un halo de inhibición de $10 \mathrm{~mm}$ y un valor de CIM de $50 \mu \mathrm{L} /$ $\mathrm{mL}$ (Tabla 2). Este resultado revela que el aceite esencial de esta especie vegetal posee buena actividad contra este microorganismo, este efecto podría adjudicarse a los componentes $\alpha$-pineno (13.57 \%) y $\beta$-pineno (3.50 \%). Al respecto, Magiatis (1999) y Filipowicz (2003) determinaron que los compuestos $\alpha$-pineno, $\beta$-pineno y limoneno presentan actividad antibacteriana al ejercer efectos tóxicos sobre la membrana, debido a la interrupción del transporte de iones y la respiración, contra este tipo de microorganismos. Esta actividad ubica al aceite esencial obtenido de las hojas de L. neriifolius como una fuente natural para la investigación de nuevas moléculas a cargo de la industria farmacéutica y lograr un tratamiento eficiente de infecciones ocasionadas por $S$. aureus, bacteria que representa un grave problema de salud pública sobre todo a nivel hospi-

Tabla 2. Actividad antimicrobiana del aceite esencial de las hojas de Libanothamnus neriifolius (B. ex H) Ernst.

\begin{tabular}{|c|c|c|c|c|c|c|c|c|c|}
\hline \multirow{3}{*}{ Microorganismos } & \multicolumn{8}{|c|}{ Zona de Inhibición (mm)* } & \multirow{3}{*}{$\underset{\mu \mathrm{L} / \mathrm{mL}}{\mathrm{CIM}}$} \\
\hline & \multicolumn{8}{|c|}{ Control Positivo } & \\
\hline & $\mathrm{AE}$ & $\mathrm{P}$ & VA & $\mathrm{NN}$ & CXM & FEP & FLU & VOR & \\
\hline Staphylococcus aureus ATCC 25923 & $10 *$ & $37^{*}$ & & & & & & & 50 \\
\hline Enterococcus faecalis ATCC 29212 & NA & & $18^{*}$ & & & & & & NE \\
\hline Escherichia coli ATCC 25922 & NA & & & $26^{*}$ & & & & & NE \\
\hline Klebsiella pneumoniae ATCC 23357 & NA & & & & $22^{*}$ & & & & $\mathrm{NE}$ \\
\hline Pseudomonas aeruginosa ATCC 27853 & NA & & & & & $30 *$ & & & NE \\
\hline Candida albicans CDC B-385 & $8^{*}$ & & & & & & $50 *$ & & 700 \\
\hline Candida krusei ATCC 6258 & $10^{*}$ & & & & & & & $20^{*}$ & 500 \\
\hline
\end{tabular}

AE: Aceite esencial; P: Penicilina ${ }^{\circledR}(10 \mathrm{U})$; VA: Vancomicina ${ }^{\circledR}(30 \mu \mathrm{g}) ; \mathrm{NN}$ : Tobramicina ${ }^{\circledR}(10 \mu \mathrm{g})$; CXM: Cefuroxima ${ }^{\circledR}(30 \mu \mathrm{g}) ;$ FEP: Cefepima ${ }^{\circledR}(30 \mu \mathrm{g}) ;$ FLU: Fluconazol ${ }^{\circledast}(100 \mu \mathrm{g})$; VOR: Vorcum ${ }^{\circledR}$ (Voriconazol) $(400 \mu \mathrm{g} / \mathrm{mL})$; NA: No activo, NE: no ensayado.

*Zona de inhibición en mm, diámetro del disco $6 \mathrm{~mm}$, media tomada de dos ensayos consecutivos. CIM: Concentración inhibitoria mínima, rango de concentración 10-900 $\mathrm{LL} / \mathrm{mL}$. 
talario, debido a la resistencia que expresa a los antibióticos comerciales (Armas et al. 2015, Gómez-Gamboa et al. 2016, Saba et al. 2017).

Solo se han realizado estudios sobre identificación de componentes volátiles y actividad antimicrobiana en especies de la Subtribu Espeletiinae, destacando la actividad frente a bacterias grampositivas como lo describen Alarcón et al. (2015), quienes señalan para Ruilopezia bracteosa (Standl.) Cuatrec., tiene actividad contra $S$. aureus y Enterococcus faecalis, con una CIM entre 100 y 600 $\mu \mathrm{g} / \mathrm{mL}$, respectivamente. De igual manera, en el estudio de la composición química y actividad antibacteriana del aceite esencial de Espeletia nana Cuatrec (Standl.), señalan actividad antibacteriana contra $S$. aureus y E. faecalis, con una CIM entre 100-600 $\mu \mathrm{g} / \mathrm{mL}$. La identificación de compuestos como $\alpha$-pineno y $\beta$-pineno, confirma la actividad antibacteriana en los aceites esenciales de estas plantas (Magiatis 1999, Filipowicz 2003).

El aceite esencial obtenido de las hojas de L. neriifolius, fue activo contra ambas levaduras ensayadas, con valores de CIM de $700 \mu \mathrm{L} / \mathrm{mL}$ para Candida albicans y de $500 \mu \mathrm{L} / \mathrm{mL}$ para C. krusei (Tabla 2). Resalta la actividad frente a $C$. krusei, agente etiológico de importantes infecciones fúngicas y que expresa resistencia natural al fluconazol, antimicótico de elección para tratar la candidiasis (Sanabria et al. 2014). De acuerdo con la literatura consultada, este es el primer estudio sobre actividad antifúngica del aceite esencial obtenido de las hojas de L. neriifolius. Aunque, se han realizado investigaciones sobre la actividad antifúngica en otras especie de Espeletiinae, así, Cordero et al. (2017) evaluaron la actividad antifúngica del aceite esencial de Coespeletia moritziana Sch. Bip., Espeletia Schultzii Wedd., y Coespeletia timotensis Cuatrecasas., encontrando actividad de C. moritziana y C. timotensis contra C. albicans con una CIM entre 200 y $600 \mu \mathrm{L} / \mathrm{mL}$ y contra $C$. krusei con una CIM entre 100 y $600 \mu \mathrm{L} / \mathrm{mL}$. Con base en estos resultados, se puede inferir que la presencia del $\beta$-felandreno como compuesto mayoritario en $C$. moritziana y $C$. timotensis, al igual que en el aceite esencial de las hojas L. neriifolius, o el sinergismo entre los compuestos identificados, en su mayoría hidrocarburos monoterpénicos, podrian guardar relación con la actividad antifúngica. Actualmente no se han estandarizado criterios para definir la actividad antimicótica de productos naturales como los aceites esenciales (Zapata 2010). Al respecto, Holetz et al. (2002) establecieron los siguientes parámetros para establecer la actividad antimicrobiana de extractos hidroalcohólicos de la siguiente manera con base a la CIM: actividad antimicrobiana buena si el valor de CIM era menor de $100 \mu \mathrm{g} /$ $\mathrm{mL}$, moderada de 100 a $500 \mu \mathrm{g} / \mathrm{mL}$ y débil de 500 a 1000 $\mu \mathrm{g} / \mathrm{mL}$, inactivo valor superior a $1000 \mu \mathrm{g} / \mathrm{mL}$. Tomando en cuenta la mencionada categorización se considera la actividad antifúngica débil para $C$. albicans (CIM de 700 $\mu \mathrm{L} / \mathrm{mL}$ ) y moderada para C. krusei (CIM de $500 \mu \mathrm{L} / \mathrm{mL}$ ).

Conclusiones.- El rendimiento del aceite esencial obtenido por el método de hidrodestilación con trampa de Clevenger a partir de las hojas frescas de L. neriifolius fue del $0.087 \%$. El análisis del aceite esencial de las hojas de L. neriifolius realizado por CG-EM, permitió identificar 20 compuestos, con un $87.28 \%$ de hidrocarburos monoterpénicos, siendo los principales componentes $\beta$-felandreno (29.04\%), $\alpha$-felandreno (19.86\%), $\alpha$-pineno (13.57\%) y $\alpha$-tujeno $(12.35 \%)$. El aceite esencial de las hojas de L. neriifolius inhibió el desarrollo de S. aureus, C. albicans y C. krusei con valores de CIM de 50 $\mu \mathrm{L} / \mathrm{mL}, 700 \mu \mathrm{L} / \mathrm{mL}$ y $500 \mu \mathrm{L} / \mathrm{mL}$, respectivamente, actividad que podría atribuirse al alto contenido de monoterpenos hidrocarbonados. Este es el primer reporte sobre actividad antibacteriana y antifúngica descrita para L. neriifolius y para el género Libanothamnus.

\section{Literatura citada}

Adams R. 2007. Identification of Essential Oil Components by Gas Chromatography/Mass Spectometry. 4th Ed. Illinois (USA). Allured Publishing Corporation.

Albornoz M. 1997. Medicina Tradicional Herbaria. Caracas: Instituto Farmacoterápico Latino S.A. Caracas-Venezuela, 64-73.

Alarcón L., A. Peña, J. Velasco, J.G. Baptista, L. Rojas, \& R. Aparicio, A. Usubillaga. 2015. Chemical composition and antibacterial activity of the essential oil of Ruilopezia bracteosa. Natural Product Communications. 10(4): 655-656. https://europepmc.org/abstract/ MED/25973502.

Armas F.A., B. Suárez, N. Crespo, \& A. Suárez. 2015. Resistencia de Staphylococcus aureus a la meticilina en aislamientos nosocomiales en un hospital provincial. Gaceta Médica Espirituana, 17: 80-91. Disponible en http://scielo.sld.cu/scielo.php?script=sci_arttext\&pid =S1608-89212015000300011

Badillo V. 2001. Lista actualizada de las especies de la familia Compuestas (Asteraceae) de Venezuela. Ernstia. 11(3): 147-215.

Bohlmann, F., H. Suding, J. Cuatrecasas, R. King, \& H. Robinson. 1980. New diterpenes from the subtribe Espeletiinae. Phytochemistry, 19(2):267- 271. https://doi. org/10.1016/S0031-9422(00)8

Clinical and Laboratory Standards Institute. CLSI (2016). Performance Standards for antimicrobial Susceptibility Testing supplement M100S. Wayne, PA: 26th ed.

Contreras-Moreno B.Z, J.J. Velasco, J.C Rojas., L.C Méndez., \& M.T. Celis. 2016. Antimicrobial activity of essential oil of Pimenta racemosa var. racemosa (Myrtaceae) leaves. Journal of Pharmacy \& Pharmacognosy Research, 4(6):224-230. http://jppres.com/jppres 16.147-4.6.224

Cordero de Rojas Y., C. Díaz, J. Velasco, L. Rojas-Fermín, R. Aparicio, A. Usubillaga, C. Colmenares, \& A. Carnevali. 2017. Composición Química y efecto antifúngico de los aceites esenciales de tres especies de frailejones de los Andes Venezolanos. Revista Facultad de Farmacia, Universidad Central de Venezuela, 80 (1-2): 60-67.

Cuatrecasas J. 1976. A new subtribe in the Heliantheae (Compositae) Espeletiinae. Phytologia. 35: 43-61. https:// doi.org/10.5962/bhl.part.2608

Cuatrecasas J. 1986. Speciation and radiation of the Espeletiinae in the Andes. Oxford. University Press Oxford. 267-303.

Figueiredo A.C, J.G. Barroso L.G Pedro \& J.J Scheffer. 2008. Factors affecting secondary metabolite production inplants: volatile components and essential oils. Flavour and Fragrance Journal. 23(4): 213-226. https://doi. org/10.1002/ffj.1875 
Filipowicz N., M. Kamiski J. Kurlenda \& M. Asztemborska. 2003. Antibacterial and antifungal activity of Juniper berry oil and its selected components. Phytotherapy Research. 17(3): 227-231. https://doi.org/10.1002/ptr.1110

García-Barriga H. 1975. Flora medicinal de Colombia. Bogotá: Instituto de Ciencias Naturales, Universidad Nacional de Colombia. Botánica Médica. 3, 345.

Giraldo, D., E. Baquero, A. Bermúdez, \& M. Oliveira-Miranda. 2009. Caracterización del comercio de plantas medicinales en los mercados populares de caracas, venezuela. Acta Botánica Venezuela. 32(2): 267-301. http://saber. ucv.ve/ojs/index.php/rev_abv/article/view/931.

Gobbo-Neto L. \& N.P. Lopes. 2007. Plantas medicinais: Fatores de influência no conteúdo de metabólitos secundários. Química, Nova, 30(2): 274-381. http://dx.doi. org/10.1590/S0100-40422007000200026

Holetz, F., G. L. Pessini, N.R. Sanches, D. A. García, C. Y. Nakamura, \& B.P. Filho, 2002. Screening of some plants used in the Brazilian folk medicine for the treatment of infectious diseases. Memórias do Instituto Oswaldo Cruz, Rio de Janeiro. 97(7): 1027-1031. http://dx.doi. org/10.1590/S0074-02762002000700017

Izco J. 2004. Botánica. Segunda Edición. Madrid. Editorial McGraw-Hill Internacional de España, S.A.U. 1-781.

Gómez-Gamboa L., D. Núñez-Chacín, A. Perozo-Mena, J. Bermúdez-Gonzales \& M. Marín. 2016. Staphylococcus aureus con resistencia múltiple a los antibióticos (MDR) en un Hospital de Maracaibo-Venezuela. Kasmera, 44(1): 53-65. http://produccioncientificaluz.org/index.php/kasmera/article/view/21302/0

Khouri, N., A. Usubillaga, L.B. Rojas \& F. Galarraga. 2000.Essential oil of Espeletia weddellii. Flavour and Fragrance Journal, 15(4): 263-265. https://doi.org/10.1002/10991026(200007/08)15:4<263::AID-FFJ907>3.0.CO;2-Q

Kováts E. 1958. Retentions indices aliphatischer Halogenide, Alkohole, Aldehyde und Ketone. Helvetica Chimica Acta. XLI. 1915.

López-Palacios S. 1984. Usos Médicos de Plantas Comunes. Mérida, Venezuela: Talleres Gráficos Universitarios, 241.

Lozina L. A, Boehringer S. B., \& Acosta O. 2005. Extrapolación en una forma posológica de valores obtenidos in vitro sobre actividad antifúngica del propóleo. Universidad Nacional del Noreste: Comunicaciones científicas y tecnológicas. Resumen: V-018. http://www.unne.edu. ar/unnevieja/Web/cyt/com2005/4-Veterinaria.

Magiatis P, E. Melliou, A. L. Skaltsounis, I. B. Chinou \& S. Mitaku. 1999. Chemical composition and antimicrobial activity of the essential oils of Pistacia lentiscus var. Chia. Planta Médica. 65(8): 749-752. https://doi. org/10.1055/s-2006-960856

Narváez S., M. L. Gómez, \& M. M. Martínez. (2008). Selección de bacterias con capacidad degradadora de hidrocarburos aislados a partir de sedimentos del Caribe colombiano. Boletín de Investigaciones Marinas y CosterasInvemar. 37(1): 61-75. https://doi.org/10.25268/ bimc.invemar.2008.37.1.182

Otaiza R. 1999. Breve Diccionario de Plantas Medicinales. Caracas, Venezuela: Los Libros de El Nacional. Colección Quirón. 19:247.

Saba C. K., J. K. Amenyona. \& S. W. Kpordze. (2017). Prevalence and pattern of antibiotic resistance of Staphylococcus aureus isolated from door handles and other points of contact in public hospitals in Ghana. Antimicrobial Resistance and Infection Control. 10(6): 1-6. https://doi. org/10.1186/s13756-017-0203-2
Sanabria R., M. Samudio, N. Fariña, F. Laspina, L. Figueredo de, G. Aguilar, \& C. Espínola. (2014). Perfil de susceptibilidad a anti fúngicos de aislados de Candida spp por el método de microdilución. Nuevos puntos de cortes para fluconazol. Memorias del Instituto de Investigaciones en Ciencias de la Salud. 12(1):33-40. http://revistascientificas.una.py/index.php/RIIC/article/view/33

Schnee L., (1960). Plantas comunes de Venezuela. Caracas. Facultad de Agronomía. Universidad Central de Venezuela.

Usubillaga A., R. Aparicio, M. Romero, L. B. Rojas, \& N. Khouri, (2001). Volatile constituents from the leaves of four Libanothamnus species from the Venezuelan Andes. Flavour and Fragrance Journal. 16(3): 209-211. https://doi.org/10.1002/ffj.973.

Velasco J., J. Rojas, P. Salazar, M. Rodríguez, T. Díaz, A. Morales, \& M. Rondón. (2007). Antibacterial activity of the essential oil of Lippia oreganoides against multiresistant bacterial strains of nosocomial origin. Natural Products Communication. 2(1): 85-88.

Zapata B., C. Durán, E. Stashenko, L. Betancur, \& A. Mesa. (2010). Actividad antimicótica y citotóxica de aceites esenciales de plantas de la familia Asteraceae. Revista Iberoamericana de Micología. 27(2): 101-103. https:// doi.org/10.1016/j.riam.2010.01.005

Zuluaga A., C. De Bedout, C.A Agudelo, H. Hurtado, M. Arango, A. Restrepo, \& A. González. (2010). Sensibilidad a fluconazol y voriconazol de especies de Candida aisladas de pacientes provenientes de unidades de cuidados intensivos en Medellín, Colombia (2001-2007), Revista Iberoamericana de Micología, 27(3): 125-129. https://doi.org/10.1016/j.riam.2010.04.001

Agradecimientos:

Los autores desean expresar su agradecimiento al Ing. Forestal Luis E. Gámez A. Herbario MER "Carlos Liscano" de la Facultad de Ciencias Forestales, Universidad de Los Andes; al Herbario MERF "Ruiz Terán" de la Facultad de Farmacia y Bioanálisis de la Universidad de Los Andes; al Cepario del Laboratorio de Microbiología y Parasitología de la Facultad de Farmacia y Bioanálisis de la Universidad de Los Andes. A la Universidad de Los Andes.

\section{Rol de los autores:}

RLA: realizó diseño, coordinación de la investigación y redacción del manuscrito. LRF: efectuó el análisis químico por cromatografía de gases acoplado a espectrometría de masas, cálculo de índices de Kováts y redacción. JV: diseño y ejecutó la determinación de la actividad antimicrobiana, análisis de datos y redacción. AU: realizó asesoramiento en el laboratorio y redacción. MS: efectuó la parte experimental extracción del aceite esencial y el ensayo antimicrobiano. JR: realizó asesoramiento en el laboratorio.

\section{Conflicto de intereses:}

Los autores no incurren en conflictos de intereses.

\section{Aspectos éticos / legales:}

Ministerio del Poder Popular para el Ambiente, Herbario MER de Ciencias Forestales, Permiso para la recolección de Muestras Botánicas № H-180.

\section{Fuentes de financiamiento:}

El presente trabajo se realizó gracias al financiamiento del Consejo de Desarrollo Científico, Humanístico y Tecnológico y de la Artes de la Universidad de Los Andes CDCHTA-ULA (Proyecto FA-562-14-08-B). 